J. DIFFERENTIAL GEOMETRY

66 (2004) 437-452

\title{
STRONG MARKED ISOSPECTRALITY OF AFFINE LORENTZIAN GROUPS
}

\author{
Virginie Charette \& Todd Drumm
}

\begin{abstract}
The Margulis invariant $\alpha$ is a function on $H^{1}\left(\Gamma, \mathbb{R}^{2,1}\right)$, where $\Gamma$ is a group of Lorentzian transformations acting on $\mathbb{R}^{2,1}$, that contains no elliptic elements. The spectrum of $\Gamma$ is the image of all $\gamma \in \Gamma \backslash(I d)$ under the map $\alpha$. If the underlying linear group of $\Gamma$ is fixed, Drumm and Goldman proved that the spectrum defines the translational part completely. In this note, we strengthen this result by showing that isospectrality holds for any free product of cyclic groups of given rank, up to conjugation in the group of affine transformations of $\mathbb{R}^{2,1}$, as long as it is non-radiant, and that its linear part is discrete and non-elementary. In particular, isospectrality holds when the linear part is a Schottky group.
\end{abstract}

\section{Introduction}

The Margulis invariant, $\alpha(\gamma)$, of a fixed point free affine hyperbolic transformation $\gamma$ measures oriented translation along the unique $\gamma$ invariant line in $\mathbb{A}^{2,1}$, affine $2+1$ Minkowski space. This is an important invariant, which Margulis used in $\left[\mathbf{6}^{\prime}, \mathbf{i}, \bar{i}\right]$, to prove the existence of proper actions by purely hyperbolic affine groups on $\mathbb{R}^{3}$. In a previous paper, we extended its definition to affine transformations with parabolic linear part [2].

Let $\Gamma_{0} \subset \mathrm{SO}(2,1)^{0}$. Since $\mathrm{SO}(2,1)^{0} \cong \operatorname{Isom}\left(\mathbb{H}^{2}\right)$, the group of isometries of the hyperbolic plane, an element is hyperbolic, parabolic or elliptic, depending on its fixed point set. An affine deformation of $\Gamma_{0}$ is an isomorphism into the group of affine transformations of $\mathbb{A}^{2,1}$ :

$$
\phi: \Gamma_{0} \rightarrow \operatorname{Aff}\left(\mathbb{A}^{2,1}\right)
$$

The authors would like to thank Bill Goldman for many helpful conversations. Received 11/01/2003. 
such that $\mathbb{L} \circ \phi=\Gamma_{0}$, where $\mathbb{L}$ denotes projection onto the linear part. A Schottky subgroup of $\mathrm{SO}(2,1)^{0}$ is a discrete, freely generated subgroup of $\mathrm{SO}(2,1)^{0}$ whose non-identity elements are hyperbolic. DrummGoldman [3.] proved that, given a fixed linear part $\Gamma_{0} \subset \mathrm{SO}(2,1)^{0}$ that is Schottky, the marked Margulis spectrum determines the affine deformation up to translational conjugacy:

Theorem 1.1 (Weak Isospectrality (Drumm-Goldman)). Let $\Gamma_{0}$ be a discrete, purely hyperbolic, free subgroup of $\mathrm{SO}(2,1)^{0}$, and $\phi_{1}, \phi_{2}$, a pair of affine deformations of $\Gamma_{0}$ such that, for every $\gamma \in \Gamma_{0}$ :

$$
\alpha\left(\phi_{1}(\gamma)\right)=\alpha\left(\phi_{2}(\gamma)\right) \text {. }
$$

Then $\phi_{1}=T \circ \phi_{2} \circ T^{-1}$, where $T$ is a translation.

Kim [is] generalized this result to higher dimensions.

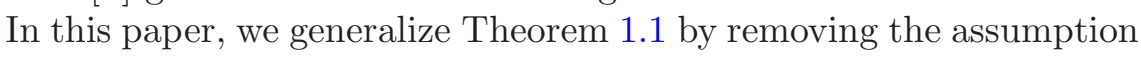
that the linear part $\Gamma_{0}$ is known. We thus consider isomorphisms from an abstract group $\mathfrak{G}$, which is assumed to be a finitely generated free product of cyclic groups, to $\operatorname{Aff}\left(\mathbb{A}^{2,1}\right)$ :

$$
\phi: \mathfrak{G} \longrightarrow \operatorname{Aff}\left(\mathbb{A}^{2,1}\right) .
$$

A group $\Gamma \in \operatorname{Aff}\left(\mathbb{A}^{2,1}\right)$ is called radiant if there is a point $x \in \mathbb{A}^{2,1}$ fixed by $\Gamma$. By Drumm-Goldman's theorem, a group freely generated by hyperbolic isometries is radiant if and only if the Margulis invariant is identically zero on $\Gamma$. We will show that if $\phi(\mathfrak{G})$ is not radiant, and if $\mathbb{L} \circ \phi$ is an isomorphism onto a discrete, non-elementary subgroup, then the growth of the Margulis invariant as a function of word length in the group determines the Aff $\left(\mathbb{A}^{2,1}\right)$-conjugacy class of $\phi(\mathfrak{G})$. (Recall that a group $G \subset \operatorname{Isom}\left(\mathbb{H}^{2}\right)$ is elementary if it admits a finite orbit.)

Theorem 1.2 (Strong isospectrality). Let $\mathfrak{G}$ be a finitely generated, free product of cyclic groups. Let $\phi_{1}$ and $\phi_{2}$ be isomorphisms:

$$
\phi_{i}: \mathfrak{G} \longrightarrow \operatorname{Aff}\left(\mathbb{A}^{2,1}\right),
$$

such that $\phi_{i}(\mathfrak{G})$ is not radiant, and $\mathbb{L} \circ \phi_{i}$ is an isomorphism onto a discrete and non-elementary subgroup of $\mathrm{SO}(2,1)^{0}$. Suppose that for every $\mathfrak{g} \in \mathfrak{G}$ such that both $\phi_{1}(\mathfrak{g})$ and $\phi_{2}(\mathfrak{g})$ are hyperbolic:

$$
\alpha\left(\phi_{1}(\mathfrak{g})\right)=\alpha\left(\phi_{2}(\mathfrak{g})\right) .
$$

Then $\phi_{1}(\mathfrak{G})$ and $\phi_{2}(\mathfrak{G})$ are $\operatorname{Aff}\left(\mathbb{A}^{2,1}\right)$-conjugate. 
This includes the case where the linear parts of $\phi_{1}(\mathfrak{G})$ and $\phi_{2}(\mathfrak{G})$ are both Schottky subgroups of $\mathrm{SO}(2,1)^{0}$.

We do not require a priori that $\phi_{1}(\mathfrak{g})$ and $\phi_{2}(\mathfrak{g})$ be of the same type (i.e., hyperbolic, parabolic or elliptic), given $\mathfrak{g} \in \mathfrak{G}$. But our assumptions do impose the following restrictions: Since $\mathbb{L} \circ \phi_{i}$ is an isomorphism onto a discrete group, $\mathfrak{g} \in \mathfrak{G}$ is of finite order if and only if $\phi_{i}(\mathfrak{g})$ has elliptic linear part. Furthermore, no two elements share common fixed points unless they are powers of each other. Finally, the fact that $\phi_{i}(\mathfrak{G})$ is non-elementary implies the existence of a hyperbolic element whose fixed point set is not invariant under the action of any elliptic element in the generating set.

The outline of the proof is as follows: Choose an appropriate generating set for $\mathfrak{G}$, so that the generating sets of both $\phi_{1}(\mathfrak{G})$ and $\phi_{2}(\mathfrak{G})$ consist entirely of hyperbolic elements.

Next, reduce to the case of rank two groups whose linear parts are Schottky. Choose appropriate conjugates of $\phi_{1}(\mathfrak{G})$ and $\phi_{2}(\mathfrak{G})$ so that the eigensystem of one corresponding pair of generators is the same, and the other corresponding pair of generators shares one eigendirection.

Equality of the Margulis invariant on hyperbolic words of the form $\eta^{n} \gamma^{m}$ determines the remaining eigendirections. We then show that the eigenvalues are also the same, thus concluding that the linear parts are equal.

By Theorem in the affine groups are thus equal up to translational conjugacy. We will include a proof of Theorem '1' '1' here for the sake of completeness.

\section{Preliminaries}

Let $\mathbb{A}^{2,1}$ denote three-dimensional affine space with the following additional structure. Its associated vector space of directions

$$
\mathbb{R}^{2,1}=\left\{p-q \mid p, q \in \mathbb{A}^{2,1}\right\}
$$

which is isomorphic to $\mathbb{R}^{3}$ as a vector space, is endowed with the standard Lorentzian scalar product:

$$
\mathbb{B}(\mathrm{x}, \mathrm{y})=x_{1} y_{1}+x_{2} y_{2}-x_{3} y_{3},
$$

where $\mathbf{x}=\left[\begin{array}{lll}x_{1} & x_{2} & x_{3}\end{array}\right]^{T}, \mathbf{y}=\left[\begin{array}{lll}y_{1} & y_{2} & y_{3}\end{array}\right]^{T} \in \mathbb{R}^{3}$. Thus, $\mathbb{A}^{2,1}$ is Minkowski $(2+1)$-spacetime. 
A non-zero vector $\mathrm{x}$ is said to be null (resp. timelike, spacelike) if $\mathbb{B}(x, x)=0$ (resp. $\mathbb{B}(x, x)<0, \mathbb{B}(x, x)>0$ ). A null vector is futurepointing if its third coordinate is positive - this corresponds to choosing a connected component of the set of timelike vectors, or a timeorientation.

The Lorentzian cross-product is the unique bilinear map:

$$
\otimes: \mathbb{R}^{2,1} \times \mathbb{R}^{2,1} \longrightarrow \mathbb{R}^{2,1}
$$

such that $\mathbb{B}(u, v \otimes w)=\operatorname{Det}([u \vee w])$. It has the following properties:

- $\mathbb{B}(\mathrm{u}, \mathrm{v} \otimes \mathrm{w})=\mathbb{B}(\mathrm{v}, \mathrm{w} \otimes \mathrm{u})$;

- $\mathbb{B}(v, v \otimes w)=0$;

- $\mathbb{B}(v \otimes w, v \otimes w)=\mathbb{B}(v, w)^{2}-\mathbb{B}(v, v) \mathbb{B}(w, w)$.

2.1. Affine deformations of a linear group. Let $\operatorname{Aff}\left(\mathbb{A}^{2,1}\right)$ denote the group of all affine transformations that preserve the Lorentzian scalar product on the space of directions. Choosing an origin in $\mathbb{A}^{2,1}$ allows one to write an affine transformation as the composition of a linear transformation with a translation:

$$
\gamma(x)=g(x)+\mathrm{v}_{g}
$$

$g$ is the linear part of $\gamma$ and $\mathbf{v}_{g}$ its translational part.

Thus, $\operatorname{Aff}\left(\mathbb{A}^{2,1}\right)$ is isomorphic to $\mathrm{O}(2,1) \ltimes \mathbb{R}^{2,1}$. Denote projection onto the linear part of an affine transformation by:

$$
\mathbb{L}: \operatorname{Aff}\left(\mathbb{A}^{2,1}\right) \rightarrow \mathrm{O}(2,1) .
$$

Let $\mathfrak{G}$ be a finitely generated group and $\phi: \mathfrak{G} \longrightarrow \operatorname{Aff}\left(\mathbb{A}^{2,1}\right)$ an isomorphism; denote the linear part of $\phi$ by $\Phi=\mathbb{L} \circ \phi$. We call $\phi$ an affine deformation of $\Phi$.

Fix the linear part $G_{\Phi}=\Phi(\mathfrak{G})$. The left action induces a $G_{\Phi}$-module structure on $\mathbb{R}^{2,1}$, which we will denote by $V_{\Phi}$. Now $\phi$ is a homomorphism if and only if the translational part satisfies the cocycle condition:

$$
\mathrm{v}_{g h}=\mathrm{v}_{g}+g\left(\mathrm{v}_{h}\right)
$$

for every $g, h \in G_{\Phi}$. Thus, an affine deformation of $\Phi$ may be interpreted as a cocycle $u \in Z^{1}\left(G_{\Phi}, V_{\Phi}\right)$, where $u(g)=\mathrm{v}_{g}$. So, we write:

$$
\phi=(\Phi, u) .
$$

Suppose now that $\psi=\tau \phi \tau^{-1}$, where $\tau \in \operatorname{Aff}\left(\mathbb{A}^{2,1}\right)$ is the pure translation: $\tau(x)=x+\mathrm{v}, \mathrm{v} \in \mathbb{R}^{2,1}$. Then, the linear part of $\psi$ is $\Phi$ and for $\mathfrak{g} \in \mathfrak{G}$, the translational part of $\psi(\mathfrak{g})$ is $u(\phi(\mathfrak{g}))+\mathfrak{v}-\Phi(\mathfrak{g})(\mathrm{v})$. In other 
words, the translational parts of $\phi(\mathfrak{g})$ and $\psi(\mathfrak{g})$ differ by the coboundary $\mathrm{v}-\Phi(\mathfrak{g})(\mathrm{v}) \in B^{1}\left(G_{\Phi}, V_{\Phi}\right)$. Thus, $H^{1}\left(G_{\Phi}, V_{\Phi}\right)$ corresponds to translational conjugacy classes of affine deformations of $\Phi$.

2.2. Hyperbolic transformations. We shall restrict our attention to those transformations whose linear parts are in $\mathrm{SO}(2,1)^{0}$, thus preserving orientation and time-orientation. The isomorphism between $\mathrm{SO}(2,1)^{0}$ and $\operatorname{Isom}\left(\mathbb{H}^{2}\right)$ gives rise to the following terminology:

Definition 2.1. Let $g \in \mathrm{SO}(2,1)^{0}$ be a non-identity element;

- $g$ is hyperbolic if it has three, distinct, real eigenvalues;

- $g$ is parabolic if its only real eigenvalue is 1 , corresponding to a one-dimensional null eigenspace;

- $g$ is elliptic if its only real eigenvalue is 1 , corresponding to a onedimensional timelike eigenspace.

We also call $\gamma \in \operatorname{Aff}\left(\mathbb{A}^{2,1}\right)$ hyperbolic (resp. parabolic, elliptic), if its linear part $\mathbb{L}(\gamma)$ is hyperbolic (resp. parabolic, elliptic). Denote by $\mathcal{H}$ the set of hyperbolic affine transformations.

Suppose $g \in \mathrm{SO}(2,1)^{0}$ is hyperbolic, with eigenvalues $\lambda_{g}, 1,1 / \lambda_{g}$, for some $0<\lambda_{g}<1$. Associated to $g$ is a null frame:

$$
\left\{\mathrm{x}^{0}(g), \mathrm{x}^{-}(g), \mathrm{x}^{+}(g)\right\},
$$

where $\mathrm{x}^{-}(g)$ (resp. $\mathrm{x}^{+}(g)$ ) is a null, future-pointing $\lambda_{g^{-} \text {-eigenvector }}$ (resp. $1 / \lambda_{g}$-eigenvector), chosen to be of unit Euclidean length, and $\mathrm{x}^{0}(g)$ is the unique 1-eigenvector such that $\mathbb{B}\left(\mathrm{x}^{0}(g), \mathrm{x}^{0}(g)\right)=1$ and $\left\{\mathrm{x}^{0}(g), \mathrm{x}^{-}(g), \mathrm{x}^{+}(g)\right\}$ is positively oriented. If $\gamma \in \mathcal{H}$, we write:

$$
\left\{x^{0}(\gamma), x^{-}(\gamma), x^{+}(\gamma)\right\}:=\left\{x^{0}(\mathbb{L}(\gamma)), x^{-}(\mathbb{L}(\gamma)), x^{+}(\mathbb{L}(\gamma))\right\}
$$

Observe that $\mathbb{B}\left(\mathrm{x}^{0}(g), \mathrm{x}^{ \pm}(g)\right)=0$, and thus $\mathrm{x}^{0}(g)$ is a positive scalar multiple of $\mathbf{x}^{-}(g) \nabla \mathbf{x}^{+}(g)$. In fact, since $\mathbb{B}\left(\mathbf{x}^{-}(g) \nabla \mathbf{x}^{+}(g), \mathbf{x}^{-}(g) \nabla \mathbf{x}^{+}(g)\right)=$ $\mathbb{B}\left(\mathbf{x}^{-}(g), \mathbf{x}^{+}(g)\right)^{2}$ :

$$
\mathbf{x}^{0}(g)=\frac{-1}{\mathbb{B}\left(\mathbf{x}^{-}(g), \mathbf{x}^{+}(g)\right)} \mathbf{x}^{-}(g) \nabla \mathbf{x}^{+}(g) .
$$

The following fact will also prove useful.

Lemma 2.2. Let $g \in \mathrm{SO}(2,1)^{0}$ be hyperbolic. Then, $\mathrm{x}^{0}(g) \otimes \mathrm{x}^{+}(g)=$ $\mathrm{x}^{+}(g)$ and $\mathrm{x}^{-}(g) \otimes \mathrm{x}^{0}(g)=\mathrm{x}^{-}(g)$. 
Proof. Since the vector $\mathrm{x}^{+}(g)$ is Lorentz-perpendicular to both itself and $\mathrm{x}^{0}(g)$, we know that $\mathrm{x}^{0}(g) \nabla \mathrm{x}^{+}(g)=k \mathrm{x}^{+}(g)$. Taking the Lorentzian inner product of both sides of the equation with $\mathrm{x}^{-}(g)$ we get:

$$
\mathbb{B}\left(\mathrm{x}^{0}(g) \nabla \mathrm{x}^{+}(g), \mathrm{x}^{-}(g)\right)=k \mathbb{B}\left(\mathrm{x}^{+}(g), \mathrm{x}^{-}(g)\right) .
$$

The left-hand side can be rearranged via a property of $\nabla$ described above:

$$
\mathbb{B}\left(\mathrm{x}^{0}(g) \otimes \mathrm{x}^{+}(g), \mathrm{x}^{-}(g)\right)=\mathbb{B}\left(\mathrm{x}^{+}(g) \otimes \mathrm{x}^{-}(g), \mathrm{x}^{0}(g)\right),
$$

Rewriting $\mathrm{x}^{+}(g) \otimes \mathrm{x}^{-}(g)$, using $\left(\begin{array}{c}\mathbf{I}_{1}^{\prime} \\ 1\end{array}\right)$, we obtain the following:

$$
\mathbb{B}\left(\mathrm{x}^{-}(g), \mathrm{x}^{+}(g)\right) \mathbb{B}\left(\mathrm{x}^{0}(g), \mathrm{x}^{0}(g)\right)=k \mathbb{B}\left(\mathrm{x}^{+}(g), \mathrm{x}^{-}(g)\right) .
$$

This implies that $k=1$. The proof for $\mathbf{x}^{-}(g)$ is similar.

q.e.d.

2.3. The Margulis invariant. Every affine hyperbolic $\gamma$ admits a unique spacelike line that is $\gamma$-invariant, denoted $C_{\gamma}$. Furthermore, $C_{\gamma}$ is parallel to $\mathrm{x}^{0}(\gamma)$, and $\gamma$ acts by translation on $C_{\gamma}$. On the subset $\mathcal{H} \subset \operatorname{Aff}\left(\mathbb{A}^{2,1}\right)$ of hyperbolic elements, we define the Margulis invariant of $\gamma$ to be the function:

$$
\alpha: \mathcal{H} \rightarrow \mathbb{R}
$$

such that:

$$
\alpha(\gamma)=\mathbb{B}\left(\gamma(x)-x, x^{0}(\gamma)\right),
$$

where $x$ is an arbitrary point on $C_{\gamma}$, the unique $\alpha$-invariant line.

The action on $C_{\gamma}$ is given by

$$
\gamma(x)=x+\alpha(\gamma) x^{0}(\gamma)
$$

for every $x \in C_{\gamma}$.

The following are elementary consequences of the definition:

Lemma 2.3 (Properties of $\alpha$ ). Suppose $\gamma \in \mathcal{H}$;

(1) for any $x \in \mathbb{A}^{2,1}, \alpha(\gamma)=\mathbb{B}\left(\gamma(x)-x, \mathrm{x}^{0}(\gamma)\right)$;

(2) $\alpha(\gamma) \neq 0$ if and only if $\gamma$ acts freely;

(3) for any $\eta \in \operatorname{Aff}\left(\mathbb{A}^{2,1}\right), \alpha\left(\eta \gamma \eta^{-1}\right)=\alpha(\gamma)$;

(4) for any $\gamma \in \operatorname{Aff}\left(\mathbb{A}^{2,1}\right), \alpha\left(\gamma^{n}\right)=|n| \alpha(\gamma)$.

Item (2) of Lemma $\overline{2} .3$ implies that if $\phi=(\Phi, u)$ is radiant, then the Margulis invariant is identically zero on the group (Theorem i. 1. proves the converse). Any two hyperbolic elements in $\mathrm{SO}(2,1)^{0}$ with the same eigenvalues, or trace, are conjugate. Furthermore, it can be shown that 
any two hyperbolic elements in $\operatorname{Aff}\left(\mathbb{A}^{2,1}\right)$ with the same linear part and Margulis invariant are conjugate by a translation. Thus, the trace of the linear part and the value of $\alpha$ determine the conjugacy classes of hyperbolic elements in $\operatorname{Aff}\left(\mathbb{A}^{2,1}\right)$.

Since $x^{0}\left(\gamma^{-1}\right)=-x^{0}(\gamma)$, we have that $\alpha\left(\gamma^{-1}\right)=\alpha(\gamma)$. When $\gamma$ acts freely, the sign of $\alpha(\gamma)$ indicates the direction in which $\gamma$ displaces points on $C_{\gamma}$.

Suppose that $\mathfrak{G}$ is a finitely generated group, and let $\Phi: \mathfrak{G} \longrightarrow$ $\mathrm{SO}(2,1)^{0}$ be an isomorphism. Keep in mind that we will be reducing to the case where $\Phi(\mathfrak{g})$ is hyperbolic for every $\mathfrak{g} \in \mathfrak{G} \backslash(I d)$. So, we can safely ignore non-hyperbolic elements in the group. Fix an ordering on the hyperbolic elements of $\mathfrak{G} \backslash(I d),\left[\mathfrak{g}_{i}\right]_{i \in \mathbb{N}}$.

The marked Margulis spectrum is determined as follows: By Item (3) of Lemma 2.3 , two affine deformations of $\Phi$ yield the same values of $\alpha$ if they are translationally conjugate. Define the following function:

$$
\begin{aligned}
\alpha_{\Phi}: H^{1}\left(G_{\Phi}, V_{\Phi}\right) & \longrightarrow \mathbb{R}^{\mathfrak{G}} \\
{[u] } & \longmapsto\left[\mathbb{B}\left(u\left(\Phi\left(\mathfrak{g}_{i}\right)\right), \mathrm{x}^{0}\left(\Phi\left(\mathfrak{g}_{i}\right)\right)\right)\right]_{i \in \mathbb{N}} .
\end{aligned}
$$

The marked Margulis spectrum of an affine deformation $\phi=(\Phi, u)$ is the image $\alpha_{\Phi}([u])$.

\section{Step one: Reduction to a simple case}

In this section, we show how to reduce Theorem 1.21 to a simpler case. Indeed, we may assume that our groups sharing a marked Margulis spectrum are rank-two groups generated by hyperbolic isometries and that their generators admit certain common characteristics.

Let $\left\{\mathfrak{g}_{1}, \ldots, \mathfrak{g}_{n}\right\}$ be a generating set for $\mathfrak{G}$, with no relations except, possibly, $\mathfrak{g}_{j}^{m_{j}}=I d$, for some of the $j$ 's. Our first concern is for the linear parts of $\phi_{1}$ and $\phi_{2}$, so it will be simpler for now to think of $\phi_{1}$ and $\phi_{2}$ as representations into $\operatorname{Isom}\left(\mathbb{H}^{2}\right)$. Thus, a hyperbolic element has two fixed points on the boundary of the hyperbolic plane, a parabolic element has one fixed point on the boundary of the hyperbolic plane, and an elliptic element fixes a point inside the hyperbolic plane. Two elements $g, h \in \operatorname{Isom}\left(\mathbb{H}^{2}\right)$ commute if and only if they share the same set of fixed points.

Since $\phi_{1}(\mathfrak{G})$ is non-elementary, it contains a hyperbolic element $\mathfrak{g}$; we may choose it such that its null eigenspace is not invariant under the action of any of the elliptic elements in the generating set. Replacing one 
of the generators and re-indexing if necessary, we may assume without loss of generality that $\phi_{1}\left(\mathfrak{g}_{1}\right)$ is hyperbolic.

Then $\phi_{2}\left(\mathfrak{g}_{1}\right)$ is either hyperbolic or parabolic, since it must also be of infinite order (the $\mathbb{L} \circ \phi_{i}$ 's are isomorphisms onto discrete subgroups of $\left.\mathrm{SO}(2,1)^{0}\right)$. If it happens to be parabolic, then $\phi_{2}\left(\mathfrak{g}_{1} \mathfrak{g}_{2}^{k}\right)$ is hyperbolic for large enough $|k|$ and its fixed point set is not invariant under the action of any elliptic generator. Choose $|k|$ large enough so that $\phi_{1}\left(\mathfrak{g}_{1} \mathfrak{g}_{2}^{k}\right)$ is also hyperbolic and its fixed point set is not invariant under the action of any elliptic generator. Thus, substituting $\mathfrak{g}_{1}$ for $\mathfrak{g}_{1} \mathfrak{g}_{2}^{k}$ if necessary, we may assume without loss of generality that both $\phi_{1}\left(\mathfrak{g}_{1}\right)$ and $\phi_{2}\left(\mathfrak{g}_{1}\right)$ are hyperbolic, and their respective fixed point sets are not invariant under the action of any elliptic generator.

Let $j \in\{2, \ldots, n\}$; then both $\phi_{1}\left(\mathfrak{g}_{1}^{k_{j}} \mathfrak{g}_{j}\right)$ and $\phi_{2}\left(\mathfrak{g}_{1}^{k_{j}} \mathfrak{g}_{j}\right)$ are hyperbolic for large enough $\left|k_{j}\right|$. Substituting if necessary, we may thus assume that for $i=1,2, \phi_{i}(\mathfrak{G})$ is freely generated by hyperbolic elements $\phi_{i}\left(\mathfrak{g}_{1}\right), \ldots, \phi_{i}\left(\mathfrak{g}_{n}\right)$.

Next, the following result allows us to further reduce to the case of a rank two group. Recall that $G \subset \operatorname{Isom}\left(\mathbb{H}^{2}\right)$ is a Schottky subgroup of $\operatorname{Isom}\left(\mathbb{H}^{2}\right)$ if it admits generators $g_{1}, \ldots g_{n}$, called Schottky generators, such that the following holds: there exist $2 n$ disjoint closed intervals $A_{i}^{ \pm}$ on the boundary of the hyperbolic plane, with $g_{i}\left(A_{i}^{-}\right)=\operatorname{cl}\left(\partial \mathbb{H}^{2} \backslash A_{i}^{+}\right)$, $i=1, \ldots, n$, where $c l$ denotes closure. In particular, if $w=g_{i_{1}}^{j_{1}} \cdots g_{i_{k}}^{j_{k}}$, $j_{k} \in \mathbb{Z}$, is reduced (i.e., $g_{i_{m+1}} \neq g_{i_{m}}, m=1, \ldots, k-1$ ), then $\mathrm{x}^{+}(w) \in$ $A_{i_{1}}^{\sigma\left(j_{1}\right)}$ and $\mathrm{x}^{-}(w) \in A_{i_{k}}^{-\sigma\left(j_{k}\right)}$, where $\sigma(j)$ is the sign of $j$.

Proposition 3.1 (Rank-two free subgroups suffice). Let $\mathfrak{G}$ be a finitely generated free product of cyclic groups, with generating set $\mathfrak{g}_{1}, \ldots$, $\mathfrak{g}_{n}$. Suppose $\phi_{1}, \phi_{2}: \mathfrak{G} \longrightarrow \operatorname{Aff}\left(\mathbb{A}^{2,1}\right)$ are isomorphisms, such that each $\mathbb{L} \circ \phi_{i}$ is an isomorphism onto a discrete subgroup of $\operatorname{Isom}\left(\mathbb{H}^{2}\right)$ and for $i=1,2, \phi_{i}(\mathfrak{G})$ is freely generated by hyperbolic elements $\phi_{i}\left(\mathfrak{g}_{1}\right), \ldots$, $\phi_{i}\left(\mathfrak{g}_{n}\right)$. Suppose, furthermore, that for every rank two subgroup, $H=$ $\left\langle\mathfrak{g}_{i}, \mathfrak{g}_{j}\right\rangle \subset \mathfrak{G}$, the restriction of $\phi_{1} \circ \phi_{2}^{-1}$ to $\phi_{2}(H)$ extends to an inner automorphism of $\operatorname{Aff}\left(\mathbb{A}^{2,1}\right)$. Then $\phi_{1} \circ \phi_{2}^{-1}$ extends to an inner automorphism of $\operatorname{Aff}\left(\mathbb{A}^{2,1}\right)$.

Proof. The result is clear if the linear parts are the same, so assume that $\phi_{1}(\mathfrak{G}), \phi_{2}(\mathfrak{G}) \subset \mathrm{SO}(2,1)^{0} \cong \operatorname{Isom}\left(\mathbb{H}^{2}\right)$. Also, we assume that $n=3$; the general result is obtained by induction. 
Suppose $G=\langle g, h, i\rangle$ and $G^{\prime}=\left\langle g^{\prime}, h^{\prime}, i^{\prime}\right\rangle$ are discrete subgroups of $\operatorname{Isom}\left(\mathbb{H}^{2}\right)$, with hyperbolic generators, such that:

$$
\begin{aligned}
\left\langle g^{\prime}, h^{\prime}\right\rangle & =\rho\langle g, h\rangle \rho^{-1}, \\
\left\langle g^{\prime}, i^{\prime}\right\rangle & =\psi\langle g, i\rangle \psi^{-1}, \\
\left\langle h^{\prime}, i^{\prime}\right\rangle & =\pi\langle h, i\rangle \pi^{-1},
\end{aligned}
$$

for some $\rho, \psi, \pi \in \mathrm{SO}(2,1)^{0}$. We may further assume that $g, h, i$ (resp. $\left.g^{\prime}, h^{\prime}, i^{\prime}\right)$ are Schottky generators for $G$ (resp. $G^{\prime}$ ). Indeed, $g, h, i$ must have distinct fixed point sets; we get Schottky subgroups by substituting for high enough powers of the generators, without affecting $\rho, \psi$ and $\pi$. Then $\psi^{-1} \rho g \rho^{-1} \psi=g$, i.e., $\psi^{-1} \rho=\zeta_{g}$, where $\zeta_{g}$ commutes with $g$ and thus admits the same null frame. Also:

$$
\begin{aligned}
\left\langle g^{\prime}, h^{\prime}\right\rangle & =\rho\langle g, h\rangle \rho^{-1}, \\
\left\langle g^{\prime}, i^{\prime}\right\rangle & =\rho \zeta_{g}\langle g, i\rangle \zeta_{g}^{-1} \rho^{-1}, \\
\left\langle h^{\prime}, i^{\prime}\right\rangle & =\rho \zeta_{h}\langle h, i\rangle \zeta_{h}^{-1} \rho^{-1},
\end{aligned}
$$

for some $\zeta_{h}$ commuting with $h$. But then $\zeta_{g} \zeta_{h}^{-1}$ commutes with $i$, implying that they share the same null frame. This is impossible: $\phi_{2}(\mathfrak{G})$ being a Schottky group, there are pairwise disjoint sets of future-pointing null vectors, one containing the attracting eigenvectors of both $g$ and $\zeta_{g} \zeta_{h}^{-1}$, and the other containing the attracting eigenvector of $i$ q.e.d.

Suppose then that $\phi_{1}, \phi_{2}: \mathfrak{G} \longrightarrow \operatorname{Aff}\left(\mathbb{A}^{2,1}\right)$ are isomorphisms satisfying the conditions of Proposition 1 . Via conjugation, we may further reduce Main Theorem 1.1. to a technical restatement of the theorem, as follows. Let $\phi=\phi_{2} \circ \phi_{1}^{-\uparrow}: \phi_{1}(\mathfrak{G}) \rightarrow \phi_{2}(\mathfrak{G})$. We will use the following notation:

$$
\begin{aligned}
\Gamma=\phi_{1}(\mathfrak{G}) & =\langle\gamma, \eta\rangle, \\
\Gamma_{\phi} & =\phi_{2}(\mathfrak{G})=\left\langle\gamma_{\phi}, \eta_{\phi}\right\rangle,
\end{aligned}
$$

where $\gamma_{\phi}=\phi(\gamma)$ and $\eta_{\phi}=\phi(\eta)$. We will denote the linear part and smallest eigenvalue of $\gamma_{\phi}$ (resp. $\left.\eta_{\phi}\right)$ by $g_{\phi}$ and $\lambda_{g_{\phi}}\left(\right.$ resp. $h_{\phi}$ and $\lambda_{h_{\phi}}$ ).

Since we are interested in conjugacy classes of $\Gamma$, we can explicitly choose particularly nice representatives of the conjugacy class in order to simplify our calculations. For any hyperbolic $g$ and $h$ in $\operatorname{SO}(2,1)^{0}$ which generate a non-elementary group, and any three distinct future pointing vectors of unit Euclidean length $\mathbf{v}_{1}, \mathrm{v}_{2}, \mathrm{v}_{3}$, there is an element $f \in \mathrm{O}(2,1)$ 
such that $\mathrm{x}^{+}\left(f g f^{-1}\right)=\mathrm{v}_{1}, \mathrm{x}^{-}\left(f g f^{-1}\right)=\mathrm{v}_{2}$ and $\mathrm{x}^{-}\left(f h f^{-1}\right)=\mathrm{v}_{3}$. This follows directly from the fact that $\mathrm{O}(2,1)$ acts transitively on triples of distinct points on the boundary of the hyperbolic plane, and that:

$$
\frac{f\left(\mathrm{x}^{ \pm}(g)\right)}{\left\|f\left(\mathrm{x}^{ \pm}(g)\right)\right\|}=\mathrm{x}^{ \pm}\left(f g f^{-1}\right) \text {. }
$$

Accordingly, conjugate $\Gamma_{\phi}$ by $f$ so that:

$$
\begin{aligned}
& \mathrm{x}^{+}(\eta)=\mathrm{x}^{+}\left(\eta_{\phi}\right), \\
& \mathrm{x}^{-}(\eta)=\mathrm{x}^{-}\left(\eta_{\phi}\right), \\
& \mathrm{x}^{-}(\gamma)=\mathrm{x}^{-}\left(\gamma_{\phi}\right) .
\end{aligned}
$$

In particular, the linear part of $h$ is determined up to a choice of eigenvalue $\lambda_{h}$.

Finally, conjugating by a pure translation translates the invariant line of a Lorentzian transformation. We may already assume that the invariant lines $C_{\eta}$ and $C_{\eta_{\phi}}$ are parallel. Further conjugate $\Gamma_{\phi}$ by a pure translation taking $C_{\eta_{\phi}}$ to $C_{\eta}$. The translational part of an isometry is determined by the displacement factor along its invariant line, which is equal to the Margulis invariant. Thus, if $\alpha(\eta)=\alpha\left(\eta_{\phi}\right)$, the translational parts of $\eta$ and $\eta_{\phi}$ are equal.

Consequently, it suffices to prove the following restatement of our Main Theorem 1.21.

Theorem 3.2 (Technical version of strong isospectrality). Let $\Gamma=$ $\langle\gamma, \eta\rangle, \Gamma_{\phi}=\left\langle\gamma_{\phi}, \eta_{\phi}\right\rangle \subset \operatorname{Aff}\left(\mathbb{A}^{2,1}\right)$ be non-radiant groups with respective linear parts $G_{I d}$ and $G_{\phi}$, such that the generators are all hyperbolic. Denote by $u, u_{\phi}$, the corresponding cocycles in $Z^{1}\left(G_{I d}, \mathbb{R}^{2,1}\right), Z^{1}\left(G_{\phi}, \mathbb{R}^{2,1}\right)$, respectively. Let $\phi: \Gamma \rightarrow \Gamma_{\phi}$ be an isomorphism such that $\phi(\gamma)=\gamma_{\phi}$ and $\phi(\eta)=\eta_{\phi}$. Suppose that:

$$
\begin{aligned}
\mathrm{x}^{+}(\eta) & =\mathrm{x}^{+}\left(\eta_{\phi}\right), \\
\mathrm{x}^{-}(\eta) & =\mathrm{x}^{-}\left(\eta_{\phi}\right), \\
C_{\eta} & =C_{\eta_{\phi}}, \\
\mathrm{x}^{-}(\gamma) & =\mathrm{x}^{-}\left(\gamma_{\phi}\right),
\end{aligned}
$$

and furthermore, that the marked Margulis spectra are equal:

$$
\alpha_{I d}([u])=\alpha_{\mathbb{L}(\phi)}\left(\left[u_{\phi}\right]\right),
$$

where $\Gamma \backslash I d$ and $\Gamma_{\phi} \backslash I d$ are given compatible orderings. Then, $\phi$ is a pure translation. 
The theorem follows from Lemma end of Section

\section{Step two: Estimates for the rate of convergence of the eigenvectors}

We first need to establish a few technical lemmas.

Let $d(\mathrm{v}, \mathrm{w})=\|\mathrm{v}-\mathrm{w}\|$ denote the Euclidean distance between the endpoints of $v$ and $w$ emanating from the origin. As $x^{ \pm}(\omega)$ are normalized to have Euclidean length 1 , we define $\mathcal{U}$ to be the set of future pointing Euclidean unit vectors. For any given $g \in \mathrm{SO}(2,1)^{0}$, we define an associated map $\tilde{g}: \mathcal{U} \rightarrow \mathcal{U}$ defined as follows:

$$
\tilde{g}(\mathrm{v})=\frac{g(\mathrm{v})}{\|g(\mathrm{v})\|} .
$$

Lemma 4.1. Given any hyperbolic element $g \in \mathrm{SO}(2,1)^{0}$ and any $\epsilon>0$, there exists an $N$ such that for all $n \geq N$, if $\vee \in \mathcal{U}$ such that $d\left(\mathrm{v}, \mathrm{x}^{-}(g)\right)>\epsilon$, then $\tilde{g}^{n}(\mathrm{v}) \in \mathcal{U}$ satisfies $d\left(\tilde{g^{n}}(\mathrm{v}), \mathrm{x}^{+}(g)\right)<\epsilon$.

This lemma is a crude, but particularly clean, version of a description of the action of a hyperbolic element on null vectors. For vectors outside the $\epsilon$-neighborhood of $\mathrm{x}^{-}(g)$, we have the following lemma about how fast the vectors approach $\mathrm{x}^{+}(g)$ :

Lemma 4.2. If $g \in \mathrm{SO}(2,1)^{0}$ is hyperbolic and $\vee \in \mathcal{U} \backslash\left\{\mathrm{x}^{-}(g)\right\}$, then $d\left(\tilde{g}^{n}(\mathrm{v}), \mathrm{x}^{+}(g)\right) \sim O\left(\lambda_{g}^{n}\right)$.

Proof. Given any distinct $\mathbf{x}^{-}(g), \mathbf{x}^{+}(g)$, and $\mathbf{v}$ there is a fixed $h \in$ $\mathrm{SO}(2,1)^{0}$ such that for $g_{c}=h g h^{-1}$, we have the following:

$$
\mathrm{x}^{-}\left(g_{c}\right)=\left[\begin{array}{l}
0 \\
\beta \\
\beta
\end{array}\right], \quad \mathrm{x}^{+}\left(g_{c}\right)=\left[\begin{array}{c}
0 \\
-\beta \\
\beta
\end{array}\right], \quad \mathrm{v}_{c}=\left[\begin{array}{l}
\beta \\
0 \\
\beta
\end{array}\right],
$$

where $\beta=\sqrt{2} / 2$ and $\mathrm{v}_{c}=\tilde{h}(\mathrm{v})$. The map $\tilde{h}$ changes distances between points on $\mathcal{U}$ by a bounded multiple. But conjugation by $h$ does not affect the eigenvalues, i.e., $\lambda_{g_{c}}=\lambda_{g}$.

Therefore, it is enough to show this lemma in the special case where the vectors are given above. By direct calculation, we have the following:

$$
d\left(\mathrm{x}^{+}\left(g_{c}\right), \tilde{g}_{c}^{n}\left(\mathrm{v}_{c}\right)\right)=\lambda_{g}^{n} \frac{\sqrt{1+\lambda_{g}^{2 n}}}{\beta\left(1+\lambda_{g}^{2 n}\right)} .
$$


Now, fix a hyperbolic isometry $g \in \mathrm{SO}(2,1)^{0}$; given another hyperbolic isometry $h \in \mathrm{SO}(2,1)^{0}$, we assert that the distance between their respective fixed eigenvectors is of the same order as the distance between their expanding and contracting eigenvectors. In other words, if we let $h$ vary so that its expanding and contracting eigenvectors approach those of $g$, the distance between $\mathrm{x}^{0}(g)$ and $\mathrm{x}^{0}(h)$ also decreases.

Lemma 4.3. Given a fixed hyperbolic element $g \in \mathrm{SO}(2,1)^{0}$ and another hyperbolic element $h \in \mathrm{SO}(2,1)^{0}$, then

$$
d\left(\mathrm{x}^{0}(g), \mathrm{x}^{0}(h)\right) \sim O\left(\max \left(d\left(\mathrm{x}^{-}(g), \mathrm{x}^{-}(h)\right), d\left(\mathrm{x}^{+}(g), \mathrm{x}^{+}(h)\right)\right)\right) .
$$

Proof. It is enough to show this lemma when one pair of expanding or contracting eigenvectors for $g$ and $h$ are the same. That is, let

$\mathbf{x}^{-}(g)=\mathrm{x}^{-}(h)=\left[\begin{array}{l}0 \\ \beta \\ \beta\end{array}\right], \quad \mathbf{x}^{+}(g)=\left[\begin{array}{c}0 \\ -\beta \\ \beta\end{array}\right], \quad$ and $\quad \mathbf{x}^{+}(h)=\left[\begin{array}{c}\beta \sin \delta \\ -\beta \cos \delta \\ \beta\end{array}\right]$.

By direct calculation

$d\left(\mathrm{x}^{+}(g), \mathrm{x}^{+}(h)\right)=\sqrt{1-\cos \delta}$ and $d\left(\mathrm{x}^{0}(g), \mathrm{x}^{0}(h)\right)=\sqrt{2}|\sin \delta| /(1+\cos \delta)$.

Then:

$$
\lim _{\delta \rightarrow 0} \frac{\sqrt{1-\cos \delta}}{(\sqrt{2}|\sin \delta|) /(1+\cos \delta)}=\lim _{\delta \rightarrow 0} \frac{\beta|\sin \delta|}{\beta|\sin \delta|}=1 .
$$

q.e.d.

\section{Step three: Equality of the eigenvectors and eigenvalues of the generators}

At this time, we can attack the main lemma needed to prove Theorem 1 . Recall that the linear part of $\gamma\left(\right.$ resp. $\left.\eta, \gamma_{\phi}, \eta_{\phi}\right)$ is denoted $g$ (resp. $\left.\vec{h}, g_{\phi}, h_{\phi}\right)$. Also we are assuming that $\mathrm{x}^{ \pm}\left(\eta_{\phi}\right)=\mathrm{x}^{ \pm}(\eta)$ and that $\mathbf{x}^{-}\left(\gamma_{\phi}\right)=\mathbf{x}^{-}(\gamma)$.

We need only consider those words of the form $\eta^{n} \gamma^{m}$ and $\eta_{\phi}^{n} \gamma_{\phi}^{m}$, $n, m \geq 0$. (Similar calculations were done by Goldman [4] , and the first author [i].].) We may assume that these words are all hyperbolic, for large enough $m, n$. Define:

$$
\mathrm{x}^{0}(g, h)=\frac{-1}{\mathbb{B}\left(\mathrm{x}^{-}(g), \mathrm{x}^{+}(h)\right)} \mathbf{x}^{-}(g) \nabla \mathrm{x}^{+}(h) .
$$


This is a unit-spacelike vector to which both $\mathrm{x}^{0}\left(h^{n} g^{m}\right)$ and $\mathrm{x}^{0}\left(h_{\phi}^{n} g_{\phi}^{m}\right)$ converge, as $n, m \rightarrow \infty$. In fact, by Lemmas $\overline{4} .2$ and 4.3 , as $n, m \rightarrow \infty$, the distance between $\mathrm{x}^{0}\left(h^{n} g^{m}\right)$ and $\mathrm{x}^{0}(g, h)$ decreases as $\max \left(\lambda_{h}^{n}, \lambda_{g}^{m}\right)$, and the distance between $\mathrm{x}^{0}\left(h_{\phi}^{n} g_{\phi}^{m}\right)$ and $\mathrm{x}^{0}(g, h)$ decreases as $\max \left(\lambda_{h_{\phi}}^{n}, \lambda_{g_{\phi}}^{m}\right)$.

Lemma 5.1. If the hypotheses in Theorem are satisfied, then $\mathbf{x}^{+}(\gamma)=\mathbf{x}^{+}\left(\gamma_{\phi}\right)$.

Proof. For any hyperbolic $\omega \in \operatorname{Aff}\left(\mathbb{A}^{2,1}\right)$, we define $E^{ \pm}(\omega)$ to be the plane containing $C_{\omega}$ and parallel to $\mathrm{x}^{ \pm}(\omega)$.

Let $q=C_{\gamma} \cap E^{-}(\eta)$ and $p_{m}=\gamma^{-m}(q)$, for $m \geq 0$. Then $q=$ $r+\kappa \mathrm{x}^{-}(h)$, for some $r \in C_{\eta}$ and $\kappa \in \mathbb{R}$. (See Figure

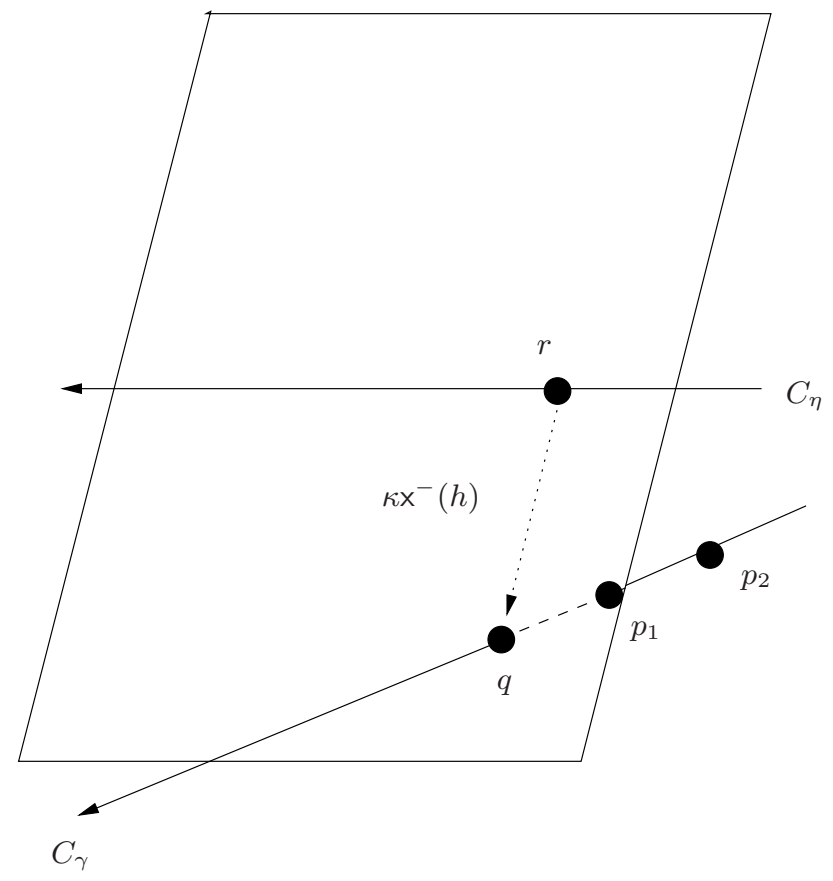

Figure 1. $q=C_{\gamma} \cap E^{-}(\eta)$ and $p_{m}=\gamma^{-m}(q), m=1,2$.

Then, $q=r+\kappa \mathrm{x}^{-}(h)$, for some $r \in C_{\eta}$ and $\kappa \in \mathbb{R}$.

We can choose similar points for $\gamma_{\phi}$ and $\eta_{\phi}$; denote by $\kappa_{\phi} \in \mathbb{R}$ the scalar corresponding to $\kappa$. We will show that $\kappa=\kappa_{\phi}$. To this end, we compute $\alpha\left(\eta^{n} \gamma^{m}\right)$ :

$$
\alpha\left(\eta^{n} \gamma^{m}\right)=\mathbb{B}\left(\eta^{n} \gamma^{m}\left(p_{m}\right)-p_{m}, \mathrm{x}^{0}\left(h^{n} g^{m}\right)\right) .
$$


Write $\eta^{n} \gamma^{m}\left(p_{m}\right)-p_{m}$ as $\eta^{n}(q)-q+q-p_{m}$. Then:

$$
\begin{aligned}
q-p_{m} & =m \alpha(\gamma) \mathrm{x}^{0}(g), \\
\eta^{n}(q)-q & =n \alpha(\eta) \mathrm{x}^{0}(h)+\kappa\left(\lambda_{h}^{n}-1\right) \mathrm{x}^{-}(h) .
\end{aligned}
$$

Since $\mathrm{x}^{0}\left(h^{n} g^{m}\right)$ converges to $\mathrm{x}^{0}(g, h)$ faster than $n$ and $m, \alpha\left(\eta^{n} \gamma^{m}\right)$ asymptotically approaches:

$$
\begin{aligned}
& m \alpha(\gamma) \mathbb{B}\left(\mathrm{x}^{0}(g), \mathrm{x}^{0}(g, h)\right)+n \alpha(\eta) \mathbb{B}\left(\mathrm{x}^{0}(h), \mathrm{x}^{0}(g, h)\right) \\
& \quad+\kappa\left(\lambda_{h}^{n}-1\right) \mathbb{B}\left(\mathrm{x}^{-}(h), \mathrm{x}^{0}(g, h)\right) .
\end{aligned}
$$

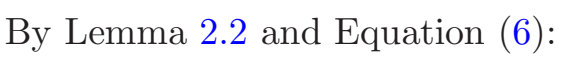

$$
\begin{aligned}
\mathbb{B}\left(\mathrm{x}^{0}(g), \mathrm{x}^{0}(g, h)\right) & =\frac{-1}{\mathbb{B}\left(\mathrm{x}^{-}(g), \mathrm{x}^{+}(h)\right)} \mathbb{B}\left(\mathrm{x}^{0}(g), \mathrm{x}^{-}(g) \nabla \mathrm{x}^{+}(h)\right) \\
& =\frac{-1}{\mathbb{B}\left(\mathrm{x}^{-}(g), \mathrm{x}^{+}(h)\right)} \mathbb{B}\left(\mathrm{x}^{+}(h), \mathrm{x}^{0}(g) \nabla \mathrm{x}^{-}(g)\right) \\
& =\frac{1}{\mathbb{B}\left(\mathrm{x}^{-}(g), \mathrm{x}^{+}(h)\right)} \mathbb{B}\left(\mathrm{x}^{+}(h), \mathrm{x}^{-}(g)\right)=1 .
\end{aligned}
$$

Thus $\alpha\left(\eta^{n} \gamma^{m}\right)$ asymptotically approaches:

$$
m \alpha(\gamma)+n \alpha(\eta)+\kappa\left(\lambda_{h}^{n}-1\right) \mathbb{B}\left(\mathrm{x}^{-}(h), \mathrm{x}^{0}(g, h)\right),
$$

and in the same fashion, $\alpha\left(\eta_{\phi}^{n} \gamma_{\phi}^{m}\right)$ asymptotically approaches:

$$
m \alpha\left(\gamma_{\phi}\right)+n \alpha\left(\eta_{\phi}\right)+\kappa_{\phi}\left(\lambda_{\eta_{\phi}}^{n}-1\right) \mathbb{B}\left(\mathrm{x}^{-}\left(h_{\phi}\right), \mathrm{x}^{0}(g, h)\right) .
$$

Since $\alpha\left(\eta_{\phi}^{n} \gamma_{\phi}^{m}\right)=\alpha\left(\eta^{n} \gamma^{m}\right)$ for all $n, m \geq 0$, and since $\mathrm{x}^{-}\left(h_{\phi}\right)=\mathrm{x}^{-}(h)$, it follows that $\kappa=\kappa_{\phi}$, as claimed.

We have assumed that $C_{\eta_{\phi}}=C_{\eta}$; thus, $C_{\gamma}$ and $C_{\gamma_{\phi}}$ must intersect the line $C_{\eta}-\kappa \mathrm{x}^{-}(h)$.

By similar reasoning, replacing $\eta$ and $\eta_{\phi}$ with their inverses above, we know that $C_{\gamma}$ and $C_{\gamma_{\phi}}$ must also intersect the line $C_{\eta}-\kappa_{-} \mathrm{x}^{+}(h)$, where $\kappa_{-} \mathrm{x}^{+}(h)$ is a vector which points from a point on $C_{\gamma}$ to a point on $C_{\eta}$. That is, $C_{\gamma}$ and $C_{\gamma_{\phi}}$ must both lie in the plane defined by the disjoint parallel lines $C_{\eta}-\kappa \mathrm{x}^{-}(h)$ and $C_{\eta}-\kappa_{-} \mathrm{x}^{+}(h)$.

We have assumed that $\mathrm{x}^{-}(g)=\mathrm{x}^{-}\left(g_{\phi}\right)$. The planes $E_{\gamma}^{-}$and $E_{\gamma_{\phi}}^{-}$ are Lorentzian perpendicular to $\mathrm{x}^{-}(g)$ and $\mathrm{x}^{-}\left(g_{\phi}\right)$, so $E_{\gamma}^{-}$and $E_{\gamma_{\phi}}^{-}$are parallel. The lines $C_{\gamma}$ and $C_{\gamma_{\phi}}$ are the intersections of two parallel planes with one fixed plane, so they must be parallel. Alternatively, $x^{0}(\gamma)=x^{0}\left(\gamma_{\phi}\right)$ so $x^{+}(\gamma)=x^{+}\left(\gamma_{\phi}\right)$. q.e.d. 
Lemma 1 can be restated as follows.

Lemma 5.2 (Fixed point isospectrality). Consider a discrete $G \subset$ Isom $\left(\mathbb{H}^{2}\right)$ which is purely hyperbolic, i.e., all non-identity elements are hyperbolic. Let:

$$
\begin{aligned}
f: G & \longrightarrow \partial \mathbb{H}^{2} \times \partial \mathbb{H}^{2} \\
g & \longmapsto\left(\mathrm{x}^{+}(g), \mathrm{x}^{-}(g)\right),
\end{aligned}
$$

where $\mathrm{x}^{+}(g)$ (resp. $\mathrm{x}^{-}(g)$ ) is the attracting (resp. repelling) fixed point of $g$. Then $f$ completely determines the group $G$.

Proof of Theorem eigenspaces of $g$ and $g_{\phi}$ are the same, as are those of $h$ and $h_{\phi}$. Also, by hypothesis, $\mathrm{v}_{h}=\mathrm{v}_{h_{\phi}}$.

The equalities $\lambda_{\gamma} \stackrel{=}{=} \lambda_{\gamma_{\phi}}$ and $\lambda_{\eta}=\lambda_{\eta_{\phi}}$ follow immediately from Lemma,$\overline{1}_{1}^{1}$. Indeed, substituting $g h g^{-1}$ for $h$, we obtain $\mathrm{x}^{+}\left(g h g^{-1}\right)=$ $\mathrm{x}^{+}\left(g_{\phi} h_{\phi} g_{\phi}^{-1}\right)$. The fact that $\mathrm{x}^{+}\left(g h g^{-1}\right)$ is parallel to $g\left(\mathrm{x}^{+}(h)\right)$ uniquely determines $\lambda_{\gamma}$. We show that $\lambda_{h}=\lambda_{h_{\phi}}$ in a similar fashion, by considering $h g h^{-1}$.

Finally, we consider the translational parts of $\gamma$ and $\gamma_{\phi}$. The proof of Lemma 5 shows that $C_{\gamma}$ and $C_{\gamma_{\phi}}$ are parallel; as a matter of fact, since both lines intersect $C_{\eta}+\kappa \mathrm{x}^{-}(h), C_{\gamma_{\phi}}$ can be translated to $C_{\gamma}$ by a translation $\tau$ that is parallel to $\mathrm{x}^{0}(h)$. This conjugation preserves $C_{h}$, thus $\eta$. Therefore:

$$
\Gamma=\tau \Gamma_{\phi} \tau^{-1},
$$

where $\tau$ is a pure translation.

q.e.d.

\section{References}

[1] V. Charette, Proper actions of discrete groups in $2+1$ spacetime, doctoral dissertation, University of Maryland, May 2000.

[2] V. Charette \& T. Drumm, Margulis' signed Lorentzian displacement for parabolic transformations, Proc. AMS, to appear.

[3] T.A. Drumm \& W.M. Goldman, Isospectrality_of_flat Lorentz 3-manifolds, J. Differential Geom. 58 (2001) 457-465, MR 1906782.

[4] W.M. Goldman, The Margulis Invariant of Isometric Actions on Minkowski $(2+1)-S p a c e$, in 'Ergodic Theory, Geometric Rigidity and Number Theory,' 149-164, Springer-Verlag, 2002, MR 1919401, Zbl 1013.53047.

[5] I. Kim, Affine action and Margulis invariant, preprint. 
[6] G. Margulis, Free properly discontinuous groups of affine transformations, Dokl. Akad. Nauk SSSR 272 (1983) 937-940.

[7] G. Margulis, Complete affine locally flat manifolds with a free fundamental group, J. Soviet Math. 134 (1987) 129-134, MR 0741860.

DEPARTMENT OF MATHEMATICS UNIVERSITY OF MANITOBA WinNiPEG, MaNitoBA CANADA

E-mail address: charette@cc.umanitoba.ca

Department of Mathematics University of PenNSYlVANiA Philadelphia, PA

E-mail address: tad@math.upenn.edu 